\title{
Using of SVM for diagnosis of major depressive disorder
}

\author{
Abdollah Ansari ${ }^{1 *}$, Mehdi Khalili ${ }^{2}$ \\ ${ }^{I}$ M.S.Student, Department of computer and informatics, Payam Noor University, Tehran, Iran \\ ${ }^{2}$ Assistant Professor, Department of computer and informatics, Payam Noor University, Tehran, \\ *Corresponding author E-mail: aoa374@yahoo.com
}

Copyright $\odot 2015$ Abdollah Ansari, Mehdi Khalili. This is an open access article distributed under the Creative Commons Attribution License, which permits unrestricted use, distribution, and reproduction in any medium, provided the original work is properly cited.

\begin{abstract}
artificial neural networks are used extensively in diagnosis are used and the system is able to detect a variety of conditions used in this study for diagnosis of major depressive disorder, 45 parameters were considered Implementation and operation using SVM model the use of different network error checked Education.
\end{abstract}

Keywords: Major Depressive, Neural Network, SVM.

\section{Introduction}

Doctors are already a lot of information and new findings in the various branches of medical face the constantly increasing volume and density can be and sometimes they are confusing and difficult to interpret the clinical findings are. Medical science will be more successful if it can be effective methods based on clinical experience and insight combined with the powerful tool of communication and information technology. Artificial neural networks are one of the newest and most effective ways of facilities [1].depression is a mental illness that causes feelings of grief and sadness and loss of interest are continuing. Most people sometimes feel sadness, depression and grief will feel depressed and sad body's natural response to life problems and loss of interest in things and people that we are. But when this feeling of extreme sadness, hopeless, helplessness and worthlessness are more than a few days or weeks, you're suffering from depression[2].There are several types of depressive disorders, including major depression, chronic depression, bipolar depression, seasonal depression, psychotic depression, postpartum depression and substanceinduced mood disorder[3].Major depression is a term for a collection of symptoms created by the America Psychiatric Association DSM-III in 1980 to go to work and then went public[4]. Support Vector Machines are based on the concept of decision planes that define decision boundaries [5]. A decision plane is one that separates between a set of objects having different class memberships [5].

\section{History research}

In this section we discuss the work done in the field of psychiatry in article [6] dynamic properties of a random neural network model in which synapses are dynamic neuron-based, short term used for diagnosing depression. The study, neuron by neuron is a binary variable that represents the state of relaxation is determined. Also, a neural network with connections to dress up and focus, which is influenced by the dynamic synapse is designed. In this article [7], depression is a common but disturbing mental disorder is considered. on the other hand, suggests that screening and grading of depression is still done manually and biased and often continuous range of "mild to moderate "and" moderate to severe "or discrete as" mild "," moderate "or "severe" is determined. Because of the continuous spectrum does not give an accurate result, this paper has tried to differentiate depression grades more accurately the propagation neural network learning is investigated using two different lead (BPNN) and fuzzy inference system (ANFIS), respectively, by surveillance and monitoring system works. In this article [8], noting that depression is a common effort to understand different perspectives on the benefits and barriers to health practitioners and design technologies has been investigated. The aim of this study was to understand how patients and doctors in technologies designed to treat patients with 
depression. That is how the 10-member group of health professionals in an interview that a list of indicators for identifying depression using a 7-point likert scale was provided and the participants were asked to understand the usefulness of these indicators express. In this article [9], to evaluate the performance of different classification methods for classifying depressed patients from healthy individuals. For this purpose, three frequency bands (alpha, beta, theta), and the entire band of the EEG are used as features. Classification SVM model is shown using a genetic algorithm to select the feature classification accuracy of $88.6 \%$ in patients with depression achieve. in this article [10], Major depression is an intelligent remote monitor, called MADRIM is provided, the main goal is to follow the evolution of MADRIM patients during their recovery in order to understand their behavior and to prevent relapse or recurrence is. due to the considerable lack of research done on the effectiveness of using neural networks for the diagnosis of major depression And also consider the need to take advantage of neural networks in the diagnosis of mood disorders and reciprocate by expanding public-their anonymity, this paper is dedicated to providing a method for the diagnosis of major depression using neural networks, will.

\section{Simulation results of neural network models with SVM}

Fig.1 and fig. 2 chart the SVM model in MATLAB environment can also Table. 3 shows the error of the network. According to the table.1, one can say with $60 \%$ margin of error is when a network error is 0.11 , which had the lowest percentages of different training. When $40 \%$ is the percentage error when the network has the highest value and is equal to 0.28 .

Table 1: SVM Network Error

\begin{tabular}{ll}
\hline Network error & Percent of Education \\
\hline 0.28 & 40 \\
0.12 & 50 \\
0.11 & 60 \\
0.12 & 70 \\
0.22 & 80 \\
0.13 & 90 \\
\hline
\end{tabular}

Regression diagram or chart determines the response simulation is aimed to answer the y-axis and x Answer Answer simulated target. Slope of the regression is called.

Epoch mean square error compared to the same period as the show. Test and evaluation, and the red-green-blue train diagram shown in early stopping point is called the minimum estimation error is.
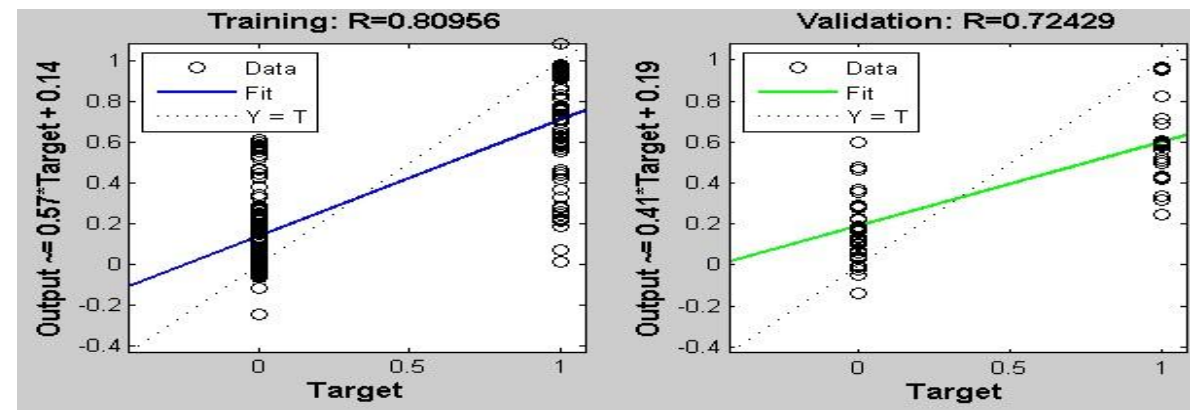

Test: $\mathbf{R}=0.53912$
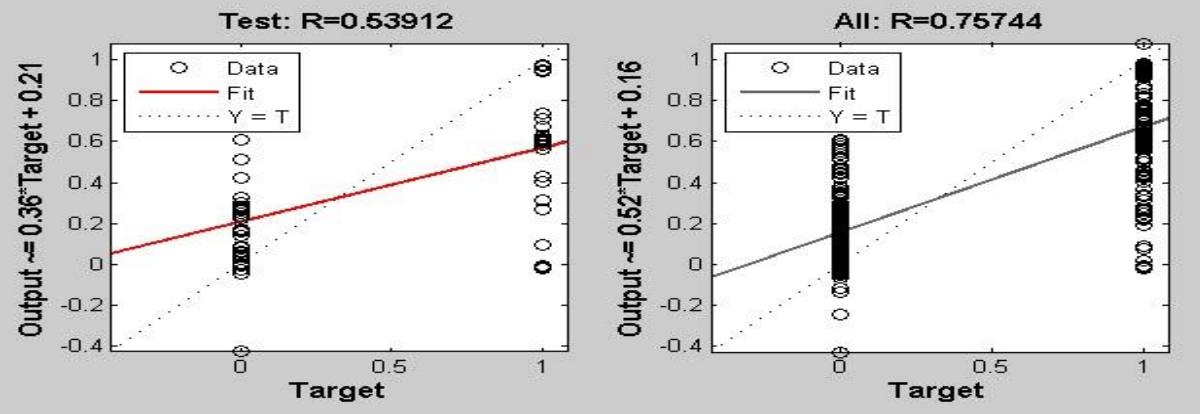

Fig. 1: Regression of SVM Model

In fig .1, the goal is to see that the horizontal lines and the vertical axis is the output of the four case study of the state of Training, the training data runs. And in Validation, with data validation runs in Test, test data runs and in the case of all, with all data runs and the $\mathrm{R}$, the closer the better.

According to the table .2, According to the table, we can see that the table shows the regression of four states studied 
Table 2: The Regression Curve for SVM Model

\begin{tabular}{ll}
\hline Regression & case \\
\hline 0.80956 & train \\
0.72429 & validation \\
0.53912 & test \\
0.75744 & all \\
\hline
\end{tabular}

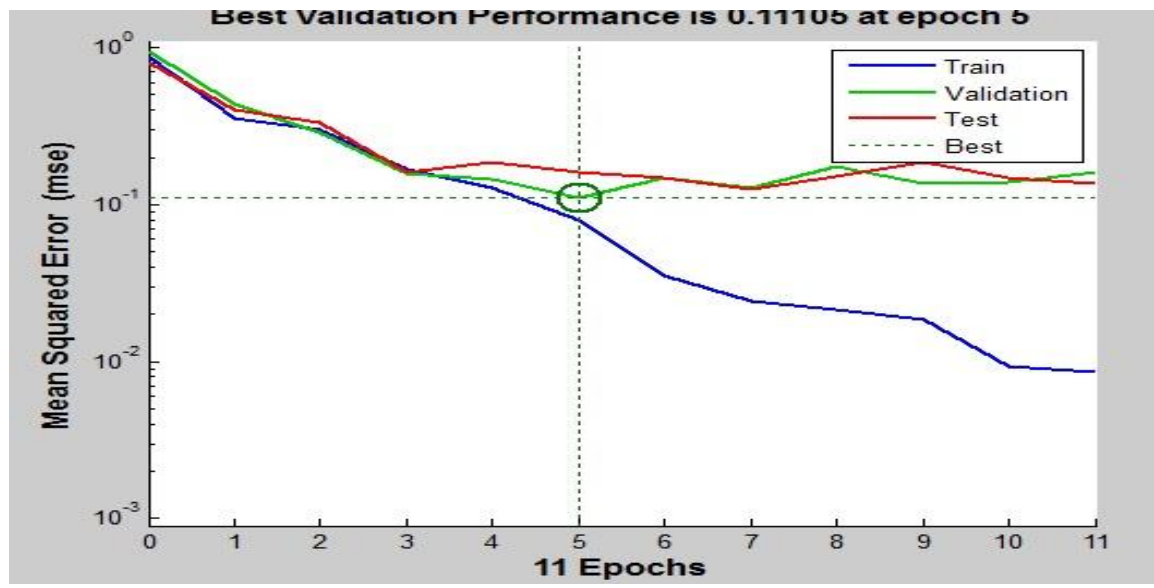

Fig. 2: Performance Graph of SVM Model

According to Fig .2, Validation the amount is equal to 0.11105 and the epoch 5 .

\section{Conclusions}

In this paper, the diagnosis of major depressive disorder after gaining 45 parameters using a neural network structures called SVM done. The accuracy rate is 0.16 equal the error.

\section{References}

[1] Allawi J, Mousavi Sh, etc,"Neural Networks and Its Application in Medicine", Journal of Govaresh, course 2, no.1, pp.49, 2007.

[2] Huerta-Ramirez R, Bertsch J, Cabello M, Roca M, Haro JM, Ayuso-Mateos JL. Diagnosis delay in first episodes of major depression: a study of primary care patients in Spain. J Affect Disord, vol.150, pp. 1247-1250, 2013. http://dx.doi.org/10.1016/j.jad.2013.06.009.

[3] Ferrari AJ, Charlson FJ, Norman RE, Patten SB, Freedman G, Murray CJ et al. Burden of depressive disorders by country, sex, age, and year: findings from the global burden of disease study 2010, PLoS Med 2013. http://dx.doi.org/10.1371/journal.pmed.1001547.

[4] Pence BW, O'Donnell JK, Gaynes BN. The depression treatment cascade in primary care: a public health perspective. Curr Psychiatry Rep, vol. 14, pp. 328-335, 2012. http://dx.doi.org/10.1007/s11920-012-0274-y.

[5] R. Cuingnet, C. Rosso, M. Chupin, S. Lehéricy, D. Dormont, H. Benali, Y. Samson and O. Colliot, Spatial regularization of SVM for the detection of diffusion alterations associated with stroke outcome, Medical Image Analysis, vol.15, pp. 729-737, 2011. http://dx.doi.org/10.1016/j.media.2011.05.007.

[6] Yuichi. Katori, Yasuhiko. Igarashi, Masato .Okada, Kazuyuki .Aihara," Stability Analysis of Stochastic Neural Network with Depression and Facilitation Synapses", no.168, pp.83-88, 2012.

[7] Chattopadhyay. S, Kaur. P, Rabhi. F, Acharya. U.R," Neural network approaches to grade adult depression Chattopadhyay", vol.36, pp. 2803$15,2012$.

[8] Kaur. P, Rabhi. F,Rajendra. Acharya,"An Automated System to Diagnose the Severity of Adult Depression", Second International Conference on Emerging Applications of Information Technology (EAIT), pp. 121 - 124, 2011

[9] Hosseinifard, Behshad, Moradi, M.H., Rostami, Reza," Classifying depression patients and normal subjects using machine learning techniques", Electrical Engineering (ICEE), 19th Iranian Conference on, pp. 1 - 4, 2011.

[10] Mugica, F. Nebot, A., Bagherpour, S., Serrano-Blanco, A.," MADRIM: A major depression remote intelligent monitor ", Fuzzy Systems (FUZZ-IEEE), IEEE International Conference on, pp. 1-8, 2012. 